\title{
LA URBANIZACIÓN EN AMÉRICA LATINA Informes de la Fundación Ford para Argentina, Chile y Venezuela (1972)
}

\author{
Urbanization in Latin America \\ Reports of the Ford Foundation for Argentina, Chile y Venezuela (1972)
}

Monti, Alejandra Inés;

(Escuela de Arquitectura, Pontificia Universidad Católica de Chile; FAPYD, Universidad Nacional de Rosario)

montialejandra@gmail.com

\section{RESUMEN}

En 1972 la Fundación Ford encarga a sus equipos consultores una serie de estudios referidos a la urbanización en los países en desarrollo titulada "Urbanization in..." (Argentina, Brasil, Chile, Colombia, Perú y Venezuela). Abordados como estudios de casos, los textos exponen el estado de situación de cada país, seleccionando los autores los temas considerados centrales para cada caso, en un universo que, desde una interpretación de las condiciones de la urbanización, pone en relieve las estructuras institucionales a escala de la planificación nacional, regional y urbana, sus niveles de institucionalización y desarrollo, así como también las referencias a los espacios formativos referidos a la enseñanza e investigación de la planificación territorial.

Palabras clave: Fundación Ford, urbanización, asistencia técnica.

Bloque temático: teoría e historia de la ciudad

\begin{abstract}
In 1972 the Ford Foundation is in charge of its consulting teams a series of studies related to urbanization in developing countries entitled "Urbanization in ..." (Argentina, Brazil, Chile, Colombia, Peru, and Venezuela). Taken as case studies, the texts expose the situation of each country, select the authors of the themes of the media topics for the case, in a universe that, from an interpretation of the conditions of urbanization, it highlights the institutional structures at the national, regional and urban planning levels, levels of institutionalization and development, as well as the references to training spaces related to the teaching and research of territorial planning.
\end{abstract}

Keywords: Ford Foundation, urbanization, technical assistance

Topic: theory and history of the city 


\section{Introducción ${ }^{1}$}

En el año 1972 la Fundación Ford (FF) encarga a John Robin y Frederick Terzo una serie de estudios referidos a la urbanización en los países en desarrollo titulada "Urbanization in..." (Argentina, Brasil, Chile, Colombia, Perú y Venezuela) en el contexto de la International Urbanization Survey. Abordados como estudios de casos, los diferentes textos exponen de manera diversa el relevamiento del estado de situación de cada país en materia de políticas territoriales, siendo tarea de los consultores la selección de los temas y tópicos discutidos. El universo temático propuesto pone en relieve las estructuras institucionales a escala de la planificación nacional, regional y urbana, sus niveles de institucionalización y desarrollo, sumado al reconocimiento de los espacios formativos referidos a la enseñanza e investigación de la planificación territorial, contribuyendo a la configuración de una síntesis que posibilita un abordaje comparativo del estado de institucionalización y desarrollo de la disciplina en el continente.

La presencia de la FF en el continente no es nueva, sin embargo a finales de la década del cincuenta se observa una redefinición de sus políticas de acción coincidente con el ciclo de expansión de la asistencia técnica y financiera tendiente a construir y transformar la agenda territorial en clave de reproducción del ideal desarrollista. El inicio de un nuevo capitulo de la política inter-continental producto de la Alianza para el Progreso (1961) va a consolidar una renovada plataforma de relaciones políticas y cruces entre los Estados Unidos y sus vecinos continentales. Esta situación es sustentada desde los organismos supranacionales articulados con la actividad de fundaciones filantrópicas extranjeras- y los propios Estados nacionales, que en cada caso van a redefinir los objetivos y las agendas en función de un ideal de progreso bajo el concepto de desarrollo.

Sostenemos que los informes de la FF de Argentina, Chile y Venezuela, ${ }^{2}$ publicados 11 años después de la firma de la Carta en Punta del Este, se configuran como exponentes del estado de situación de las políticas territoriales y los proyectos emblemáticos del llamado ciclo "desarrollista" en América del sur. Este reconocimiento, lejos de intentar un relevamiento exhaustivo de las experiencias particulares, manifiesta los límites, aciertos y fracasos de las estructuras burocráticas estatales y de las teorías e ideas referidas a las transformaciones estructurales del continente, que entendían al territorio como espacio síntesis del cambio.

Partimos de la hipótesis de que el paradigma planificador desarrollista adquirió diferentes posicionamientos y matices en cada país de la región, imposibilitando una visión homogénea del problema que asume relaciones dinámicas al interior de cada caso conjugando decisiones políticas, estructuras institucionales, capacidad de "expertos" locales y extranjeros, sumado a matrices disciplinares que si bien comparten un núcleo de pensamiento común, conforman en su aplicación (desde la investigación, la enseñanza y la praxis) diferentes grados y alcances de implementación e innovación. Desde esta perspectiva, advertimos que en los tres informes analizados se presentan condiciones diferentes del estado de la urbanización, aunque subyace una posición crítica frente a las implicancias y alcances del desarrollo en materia territorial que, sin tener la carga ideológica de la dependencia, va a identificar las condicionantes de los últimos 10 años de políticas de planificación tanto en gestión como en generación de políticas públicas que reviertan los desequilibrios presentes en la organización de los territorios en el sur del continente.

Para dar cuenta de esto, planteamos una lectura comparativa de los informes desde una perspectiva críticainterpretativa, avanzando en los puntos en común y las divergencias relevadas para cada caso, entendiendo que las mismas se inscriben dentro de un programa interno de asistencia técnica internacional de la FF que encuentra a América Latina como uno de los territorios de indagación. Dos condiciones convierten a este grupo de escritos en focos de interés: en primer lugar el intento por construir un diagnóstico detallado a nivel de "catálogo" de las experiencias referidas al proceso de urbanización y las estructuras burocráticas desarrollistas

\footnotetext{
${ }^{1}$ Este escrito ha sido realizado en el marco del Proyecto Fondecyt Postdoctoral № 3170311 "Los centros del Cono Sur y la producción de saberes en torno a la planificación y el desarrollo", del cual la autora es investigadora responsable. Se agradece a Fondecyt el financiamiento otorgado para la realización de este escrito.

${ }^{2}$ La serie de informes es parte del relevamiento realizado en los Archivos de la Fundación Ford en el marco del proyecto Fondecyt № 3170311.
} 
de cada país, independiente de las inversiones e intervención de la FF. En segundo término, se reconoce una diferencia entre el diagnóstico de Chile y sus vecinos, identificando un tono desesperanzador sobre las actuaciones de la última década en Argentina y el ciclo de expansión y ocaso venezolano a diferencia de una programa planificador sostenido en el tiempo en el país trasandino que se inicia con la modernización de la burocracia estatal propuesta por la Democracia Cristiana en $1964^{3}$ y la continuidad parcial y re-visionada del gobierno de Salvador Allende.

Advertimos que la publicación de los informes en 1972, más allá de alinearse a la encuesta internacional de urbanización, marcó un cierre de un modo de actuación en materia territorial que comenzaba a redefinir los límites del trinomio modernización-urbanización-desarrollo característico de la década del sesenta. Esta condición se agudiza drásticamente con el golpe cívico-militar chileno encabezado por Augusto Pinochet en 1973, cerrando un ciclo regional de colaboración fluida y dinámica entre la FF y los países latinoamericanos.

La ponencia plantea avanzar en el rol y los alcances de la asistencia técnica externa en América Latina a partir de una interpretación crítica anclada en las retroalimentaciones y la circulación de las ideas que permita iluminar la actividad de la FF en el continente en materia territorial. A continuación analizamos la estructura de los tres informes, a fin de identificar los puntos relevados en cada caso y las hipótesis subyacentes para cada país en relación a sus condiciones de urbanización. Por último, nos centramos en el reconocimiento de las dos experiencias de planificación llevadas a cabo por Venezuela y Chile: Ciudad Guayana y la Macrozona Central respectivamente; a fin de identificar las lecturas de las experiencias concretas y sus grados de éxito frente al objetivo principal de promoción y re-equilibrio territorial.

\section{El rol de la asistencia técnica}

En las relaciones internacionales entre los Estados Unidos y sus vecinos continentales hay temas que se presentan constantes por más de medio siglo: la idea de "dominación e intervención", "vecindad y mutualidad" $y$, con diferentes alteridades, asistencia técnica y financiera. Este recorrido de interacciones se inicia con el corolario de la Doctrina Monroe (1904), seguido por consolidación de la Pan American Union (1906), ${ }^{4}$ la Política de Buenos Vecinos (1933) y finalmente, en el contexto de la Guerra Fría, la Alianza para el Progreso (1961). Este derrotero político y económico, permite avanzar en la comprensión de la Alianza como "a program in making" (Perloff \& Resources for the Future, 1982), que alcanza en diferentes rangos y con diferentes intensidades los tres temas centrales de los vínculos intercontinentales. En los hechos, esta lectura totalizante asume en las escalas nacionales matices y transformaciones que dificultan una comprensión lineal y secuencial $y$, particularmente, sin fricciones entre los países latinoamericanos y la potencia norteamericana.

Los Estados Unidos durante la Guerra Fría identificaron a su enemigo: el comunismo y, por lo tanto, la Revolución Cubana (1959) asume un papel definitorio como contraparte de esta disputa en el territorio continental. En esta línea, si bien la política y la economía consolidan los vínculos entre norte y sur, es en la diplomacia cultural donde se invierten nuevos recursos, tanto en términos económicos como simbólicos. Pratt sostiene que es en la "llamadas zonas de contacto transnacionales donde el poder del Estado se ejerce mediante una serie de representaciones, sistemas simbólicos y nuevas tecnologías, a través de las redes de negocios y comunicaciones de las industrias culturales" (Pratt, 2011). La propia FF asume esta condición, reconociendo sólo después de la Revolución en Cuba a Latinoamérica como un área geográfica prioritaria que, como afirma Calandra, se presenta gracias al amparo de las ciencias sociales (Calandra \& Franco, 2012). Son estas las receptoras mayoritarias de los fondos de la FF en el continente, con una presencia central en la disciplina regional y urbana que se consolida a partir de la financiación a la creación de nuevos espacios académicos universitarios de formación de posgrado, los aportes a la Sociedad Interamericana de Planificación

\footnotetext{
${ }^{3}$ En 1964 asume la presiddencia de Chile Eduardo Frei Montalva como representante de la Democracia Cristiana. El lema de campaña Revolución en Libertad va a consolidar una tercera vía frente a la división del mundo de la guerra fría.

${ }^{4}$ La Pan American Union se crea en abril de 1890 bajo el nombre de Commercial Bureau of the American Republics. En la segunda conferencia internacional (1902) el nombre cambia a International Bureau of the American Republics y, en la tercera (1906) se define la Pan American Union hasta el año 1948 que va a ser denominada Organization of American States.
} 
(SIAP) para su concreción y funcionamiento, el envio de misiones y expertos a todo el continente, sumado a la asesoria a oficinas de planificación nacionales y regionales.

En el informe Urbanization in the Developing Countries: the response of International Assistance (Terzo, 1972) el encargado de la serie expone la transformación de los lineamientos de la asistencia técnica en lo referido a los abordajes sobre la urbanización como tema, reconociendo las múltiples agencias que, desde 1950, tienen a América Latina como objeto de investigación. Las referencias a los posicionamientos y políticas/programas de acción y financiamiento de las Naciones Unidas a través del Comité de Vivienda y Planificación, del Banco Central, de la Comisión Económica para América Latina (CEPAL) y de la United States Agency for International Development (USAID) y el Banco Interamericano de Desarrollo (BID) ${ }^{5}$ se constituyen como una primera aproximación a un problema que, en el continente, marcaba el ritmo de las agendas de Estado.

Lester Pearson en 1969 realiza una crítica a como se llevó adelante la asistencia técnica en América Latina, sosteniendo que la misma fue fragmentaria en relación a los planes nacionales y los "papers" de los equipos técnicos de cada país, situación que condiciono el accionar de las agencias internacionales de financiación y la consecuente participación de los equipos técnicos internacionales en los proyectos locales (Terzo, 1972). Este posicionamiento, es acompañado por la visión de Terzo que sostiene que "la asistencia técnica ha reflejado una confianza infundada en la transferencia de estándares y técnicas de planificación occidentales sin una adaptación adecuada e innovadora de estas técnicas a las condiciones particulares de los países en desarrollo. La asistencia financiera, aunque importante para las ciudades, ha sido simbólica y paliativa y se ha visto abrumada rápidamente por el ritmo del crecimiento urbano" (Terzo, 1972). A su vez, reconoce cierta abstracción en la realización de los proyectos y programas, con una fuerte impronta académica, pero sin la necesaria precisión y realidad política. Siguiendo esta línea, se plantea la inadecuada acción de las agencias internacionales en materia de asistencia técnica a los paises en desarrollo, entendido a partir de una negación a la revisión de sus mecanismos de acción durante las décadas del cincuenta y sesenta, desde un enfoque que encuentra la genesis de sus fracasos en el acelerado proceso de urbanización y no en lo inadecuado de las escalas de intervención ni en los efectos negativos que la asistencia técnica y financiera han tenido en el entorno urbano.

\section{La construcción de una serie}

El análisis de los informes permite construir una serie centrada en una perspectiva particular: la de los consultores de la FF y, por consiguiente, la mirada externa frente a un tema central de la disciplina, el proceso de urbaanización en América Latina. La lectura comparativa de los tres casos seleccionados ${ }^{6}$ posibilita avanzar en el trazado de un esquema que da cuenta, en mayor o menor medida, del ciclo desarrollista de matriz planificadora desde sus inicios hasta el principio del fin. En ocasiones mediado por las posiciones de los planificadores locales, el relevamiento de las experiencias abordan en rasgos generales tres aspectos: la configuración nacional de la urbanización y su distribución territorial; las estructuras estatales de planificación y sus proyectos concretos y, por último, los esquemas de formación de planificadores.

\subsection{Argentina}

El informe presentado por la FF respecto a la Argentina muestra la complejidad de un país a la hora de delinear sus políticas territoriales. Expuesto en 14 paginas, la encuesta se basa principalmente en la conversaciones del equipo de la FF con los integrantes del CEUR ${ }^{7}$, identificando que un informe detallado no sería útil para la encuesta de urbanización internacional (Ford Foundation, 1972). Esta inutilidad se define a partir del

\footnotetext{
${ }^{5}$ Terzo reconoce tres categorias para identificar las agencias que operan enla temática de la urbanización: las agencias multilaterales que proveen oportunidades de entrenamiento y asistencia técnica en planificación urbana, vivienda e infraestructuras; las agencias bilaterales que incorporan a lo anterior el capital para la ejecución de proyectos de viviendda e infraestructura; y por último, los bancos de desarrollo que porporcionan asistencia financiera generalmente en la temática de infraestructuras urbanas.

${ }^{6}$ La selección de los casos se vincula a las temáticas desarrolladas por el Proyecto №3170311.

${ }^{7}$ EI CEUR cuenta con financiación para su funcionamiento de la FF desde sus inicios como IPRUL en Rosario en el año 1962.
} 
reconocimiento de la falta de innovación en planificación regional y urbana, así como también en el diseño urbano, sumado a la carencia de habilidades de investigación en el tema.

En esta línea, los aportes del informe al reconocimiento de la temática en Argentina son bastantes escasos, identificando tres partes diferenciadas: a) en primer lugar, un abordaje cuantitativo de carácter demográfico, con el fin de mostrar la primacía de la ciudad de Buenos Aires y su área metropolitana frente al resto del país. Esta identificación es acompañada por el reconocimiento de la concentración de población y la consecuente actividad económica (productiva y de servicios) en el eje litoral del rio Paraná y el Rio de la Plata (Santa Fe / Paraná - La Plata), y la incapacidad de dar respuestas y planificar el crecimiento metropolitano que desde la década del cincuenta frente a un escenario caracterizado por la expansión y la localización de población migrante en el cordón circundante a la Capital Federal; b) la segunda parte, responde a la actividad del órgano de planificación más importante del país: el Consejo Nacional de Desarrollo (CONADE) ${ }^{8}$ que en 1971 propone el Plan Nacional de Desarrollo y Seguridad 1971-1975 en el que el tema urbano no es relevado, mientras que la región y la vivienda son analizados desde una perspectiva económica de marcado sesgo liberal según los integrantes del CEUR; c) la última sección refiere a la capacidad profesional del país, siendo el CEUR la única referencia analizada. Esta revisión evidencia un posicionamiento que ya venia siendo expuesto desde las filas del centro, reconociendo que a pesar de la importancia del mismo a escala latinoamericana, si incidencia en la política nacional es nula, en parte porque la Argentina no aplico acciones innovadoras en planificación regional y urbana (Ford Foundation, 1972). La aparición de esta crítica de la realidad del país, expone la centralidad de la voz del CEUR dentro del informe, en un reconocimiento del rol profesional del centro y su marcado giro a la construcción crítica a escala regional y el abandono de la praxis, ya que la Argentina va detrás de los lideres en la transformación de la escena urbana (Ford Foundation, 1972).

\subsection{Chile}

El caso de Chile es quizás el que presenta más cabalmente la articulación entre procesos de planificación y las condiciones de urbanización. El informe versa entre las políticas de planificación en sus múltiples escalas con la respectiva modernización del aparato estatal para viabilizar los procesos de planificación territorial en clave económica y social; y por otro lado, experiencias concretas vinculadas no sólo a los proyectos específicos (Macrozona Central), sino también a las condiciones particulares que convirtieron a Chile en un laboratorio de pruebas tanto para las agencias internacionales como para la elite política de la Democracia Cristiana (196469) y la Unidad Popular (1970-72).

Esto se expresa en los apartados del informe, reconociendo un espacio primordial a la política de planificación llevada adelante por el gobierno de la Unidad Popular, que abandona la idea de los polos de desarrollo en favor de los espacios económicos integrados, reconfigurando los instrumentos de planificación anterior en función de los nuevos fines y la conversión del país a una economía socialista. Estas interpretaciones son acompañadas por una exhaustiva lectura de las instituciones de planificación chilena, sumado a un relevamiento de los documentos producidos entre 1960 y 1971 en materia de planificación en el país. En la descripción del organigrama institucional de la planificación en Chile, se abordan las diferentes escalas: nacional con ODEPLAN, ${ }^{9}$ regional con ORPLAN,${ }^{10}$ y la escala urbana a partir del accionar del MINVU. ${ }^{11}$ Además de la estructura institucional, se le otorga especial relevancia a la denominada Operación Sitio, iniciada en 1965, reconociendo así un abanico institucional múltiple referido a los programas de planificación y sus alcances específicos.

Por último, interesa remarcar el reconocimiento de las agencias internacionales que actuaron en el país, con la correspondiente advertencia del cambio en la financiación y asistencia técnica desde la Democracia Cristiana (propensa al medio norteamericano) y la Unidad Popular (que consideraba intervencionista la presencia de

\footnotetext{
${ }^{8}$ El CONADE se crea por Decreto $N^{\circ} 7.290$ dispuesto por el artículo $34^{\circ}$ de la Ley $N^{\circ} 14.439$ en 1961

${ }^{9}$ Oficina de Planificación Nacional

${ }^{10}$ Oficina de Planificación Regional

${ }^{11}$ Ministerio de Vivienda y Urbanismo
} 
externos). Si el primer periodo se caracteriza por la confluencia de múltiples organismos y fundaciones que financiaron instituciones, oficinas estatales, proyectos, profesionales y la formación en el exterior de los equipos técnicos; durante el gobierno de Allende la asistencia se materializó en programas bilaterales coordinados por CORHABIT mayoritariamente.

\subsection{Venezuela}

El estudio venezolano asume una estructuración particular. En primer lugar a los autores Terzo y Robin se le suma el local Valenzuela, situación que plantea un diferencia del rol de los expertos latinoamericanos en la producción del informe, ya no como profesionales encuestados sino como productores del diagnóstico. En términos de contenido, la organización del escrito asume a la política regional como su principal baluarte, estableciendo el mayor nivel de innovación de las políticas de desarrollo regional de base industrial y a la Ciudad Guayana como la estrategia de condensación de un modelo alternativo de centro y periferia a escala nacional. A continuación de esta experiencia pionera en la materia en América Latina, identifican desde un modelo clásico de diagnóstico el caso de la capital: Caracas. La misma es reconocida como cabecera de una megalópolis emergente que posee una de las tasas de urbanización más altas de toda América Latina. Sin embargo, y a pesar del reconocimiento del proyecto de planificación urbana de Caracas, los autores trabajan sobre las contradicciones de su proceso de urbanización que pivotea entre "la reluciente opulencia de sus ahora edificios de oficinas y apartamentos de lujo y la pobreza de sus ranchos que representan el $23 \%$ de la población del área" (Robin, Terzo, \& Valenzuela Sholz, 1972: 36). A continuación se identifican las características del resto de otros centros urbanos del país, reconociendo la conformación de conglomerados urbanizados en torno a Maracaibo que concentran la actividad económica vinculada al petróleo.

La última parte del informe, refiere a las instituciones y agencias que operan en materia territorial en el país. A escala municipal se reconoce la actividad de FUNDACOMUN ${ }^{12}$ (Fundación para el desarrollo de la comunidad y el fomento municipal) cuyo rol es el de mejorar las gestiones de los gobiernos municipales y el aumento de sus recursos y habilidades a partir de la asistencia técnica. ${ }^{13} \mathrm{~A}$ partir de sus cuatro sedes administrativas, la Fundación establece un proceso de regionalización tendiente a la descentralización de los núcleos urbanos del país. Asimismo se reconoce las agencias que prestaron asistencia técnica y financiera a FUNDACOMUN como el programa USAID de manera directa y la Fundación Ford a través de la financiación al Instituto de Administración Pública. A nivel nacional, se reconoce la actividad de CORDIPLAN, la agencia de planificación nacional del país que además se encarga de los programas de vivienda, y las infraestructuras urbanas. Naciones Unidas es uno de los principales promotores de CORDIPLAN a través de la financiación de asesores técnicos en movilidad y planificación urbana para los diferentes programas. USAID en tanto centra su acción en los proyectos de vivienda de la corporación con la financiación de proyectos concretos y al Instituto Venezolano de Cooperación de la Vivienda. El Banco Interamericano de Desarrollo y el Banco Mundial financian proyectos específicos como los estudios para el sistema de metro o la instalación de infraestructuras de provisión de agua potable o tendido eléctrico.

La visión de los consultores sobre Venezuela establece la complejidad y grado de avance significativo de la organización nacional en materia de planificación y de urbanización, considerando al país como uno de los potencialmente mas desarrollados del continente. Sin embargo, a pesar de contar con una de las experiencias más complejas en materia de desarrollo regional: ciudad Guayana, los límites de la planificación, 10 años después se hacen visibles a los ojos de los consultores. Esta complejidad para llevar adelante el proceso de planificación, que contaba con el apoyo tanto del gobierno local como de los organismos y agencias internacionales, comienza a relativizar la fe en la planificación como técnica de fuerte impronta racional,

\footnotetext{
12 FUNDACOMUN fue creada por decreto presidencial en 1962, bajo la presidencia de Rómulo Betancurt.

${ }^{13}$ Las áreas de asistencia técnica de FUNDACOMUN son: servicios municipales; asistencia legal; administración fiscal, planificación urbana; catastro urbano; entrenamiento profesional; y orientación económica.
} 
identificando en las propias condiciones locales (sociales, políticas y culturales) las causas del incumplimiento de los planes previstos.

\section{La región como proyecto: Macrozona Central y Ciudad Guayana}

La MacroZona Central se constituye como un proyecto que pretende dar respuesta al crecimiento de la urbanización de 3 regiones planificadas (IV, V y la Región Metropolitana) concentrando juntas el 51\% de la población de Chile. El reconocimiento de la voluntad de regionalización del desarrollo urbano por parte del gobierno de Allende, va a marcar un cambio de las orientaciones en materia de urbanización estableciendo que el "centralismo de Santiago atenta contra la integración económica nacional" (Robin \& Terzo, 1972: 16) siendo necesario actuar por fuera del distrito metropolitano para poder descentralizar la producción a partir de una redistribución de la inversión. Los autores reconocen que en el cambio de la política de la Democracia Cristiana (sobre la base fuerte de la planificación nacional) y las propuestas del gobierno de Allende se establece un dilema ideológico de la planificación que se plantea antagónico políticamente: la visión de la asistencia técnica norteamericana frente a la mirada marxista. Territorialmente estos posicionamientos se dividen entre la concentración geográfica como atributo del mercado económico capitalista (lo cual constituye un obstáculo para la socialización del superávit) frente a un modelo que pone en conflicto las metas de eficiencia y equidad, en otras palabras centra su debate en torno al problema del centro y las periferias.

Esta perspectiva es leída por los autores a partir de los trabajos de investigación y proyectos realizados desde el CIDU, identificando en las figuras de Guillermo Geisse y José Luis Coraggio primero y Andrés Necochea y John Friedmann después, la base conceptual del encargo del gobierno al centro perteneciente a la Universidad Católica. La discusión en torno a las áreas metropolitanas es levantada en clave latinoamericana, identificando que "el verdadero problema de los países latinoamericanos es la necesidad de una restructuración social que atañe a todos los miembros de la sociedad a participar de los beneficios económicos del crecimiento económico controlado y estimulado por las fuerzas exógenas" (Robin \& Terzo, 1972: 36). Los miembros del CIDU separan el problema de la urbanización del crecimiento industrial, lo que va a articular modelos económicos diferenciados para el área metropolitana y las regiones, reconociendo una creciente "especialización" territorial en el país. Sin embargo, en el mismo momento de producción ya se identificaban los problemas de la MZC que lejos consolidarse como una unidad es "todavía una serie de puntos no relacionados con un mercado urbano no integrado" (Robin \& Terzo, 1972: 37)

El proyecto de Ciudad Guayana es reconocido por los autores como un caso emblema para toda América Latina referido a la generación de un nuevo polo de crecimiento que permita sustituir y transformar la estructura territorial nacional a partir de la generación de un nuevo sector de base industrial que redefine el modelo centro y periferia característico de los países de la región. Con la creación de un sistema de planificación nacional en 1956, Venezuela se convierte en uno de los países más innovadores en materia de planificación de la economía nacional, intentando reducir la dependencia al petróleo a partir del fomento de la industrialización y la generación de nuevas áreas de crecimiento urbano. Ciudad Guayana se convierte así en la ciudad cabecera de una nueva región económica que, desarrollada por la Corporación Venezolana Guayana, se propone como punta de lanza para el desarrollo regional a través de innovaciones en materia de energía, minería, agricultura y recursos forestales. Sin embargo Robin y Terzo, plantean el fracaso de la experiencia que, para 1971, no había logrado encauzar su crecimiento ni cumplir con las proyecciones realizadas en 1961 por los equipos técnicos del MIT ,con John Friedmann como uno de sus mayores planificadores asociados, junto a los expertos locales nucleados en el CENDES. Los autores sitúan en la mirada utilitarista de la urbanización el problema de la falta de población, identificando que el caso es un prototipo del desarrollo de una política regional que "sería una gran contribución a la economía de Venezuela, aunque el éxito de Ciudad Guayan (con los recursos y la experiencia hasta el momento) son modestos en el arranque" (Robin, Terzo, \& Valenzuela Sholz, 1972: 20) 


\section{Aproximaciones finales}

Los informes de la FF constituyen una mirada externa del estado de situación de la urbanización y la planificación en cada uno de los países. Apoyados por los técnicos locales, los relevamientos realizados por Robin y Terzo permiten avanzar en una deconstrucción de una condición monolítica de la Fundación Ford, reconociendo que si bien existieron líneas generales de financiación y asistencia técnica, es a partir de la interacción con las experiencias locales y los profesionales de cada país, que los informes adquieren características diferenciadas, redefiniendo los textos como exponentes del cruce entre la mirada externa y las realidades locales.

El caso de Argentina es quizás el más emblemático, su brevedad extrema habla más de la inestabilidad política y de políticas de planificación de un país federal en términos administrativos, pero fuertemente centralista en lo referido a su política territorial. Son los técnicos del CEUR los que desaniman a los consultores de avanzar en la investigación, evidenciando que para 1971/72 los límites de la transformación estructural que permite el despegue del país era un hecho, junto con la incapacidad de los planificadores de promover esa transformación desde la academia y el Estado.

Venezuela se presenta como una experiencia intermedia, ya que cuenta con experiencias de planificación a escala regional que promovieron la transformación de la estructura económica y por consiguiente territorial a partir de la creación de nuevas ciudades vinculadas a la actividad industrial. Los esfuerzos aquí están puestos en la medición de los límites del programa de ciudad Guayana, sumado al reconocimiento de las instituciones políticas que operan en la gerencia de la urbanización.

Chile en tanto, presenta un informe más exhaustivo de su política y experiencia de planificación. En parte, cabe recordar que el país es uno de los que recibe mayor financiación de la FF en materia territorial de América Latina. Además como epicentro de las sedes de organismos supranacionales desde finales de la década del cincuenta se caracterizo por la circulación de expertos internacionales que, acompañado de un fuerte proceso de modernización estatal, convierte a la experiencia chilena en un laboratorio de experimentación que va a adquirir una nueva dimensión con las políticas de la Unidad Popular.

El ciclo de la planificación desarrollista y sus límites son expresados en los proyectos concretos de la MacroZona Central y ciudad Guayana. Sin adscribir a la teoría de la dependencia, los consultores asumen una visión crítica sobre la realidad local, no sólo atribuida a la falta de políticas públicas o a las vicisitudes y tensiones económicas, reconociendo que parte del fracaso de los proyectos de regionalización y descentralización de la urbanización se presenta fuertemente anclado en las propias tradiciones locales, tanto políticas como culturales y sociales.

\section{BIBLIOGRAFÍA}

CALANDRA, B., \& FRANCO, M. (2012). La guerra fría cultural en América Latina: desafíos y límites para una nueva mirada de las relaciones interamericanas.

FORD FOUNDATION. (1972). Urbanization in Argentina (No. 006750) (p. 14). New York: Ford Foundation. Recuperado de Rockefeller Archive Center. (FA739C - Box 303)

PERLOFF, H. S., \& Resources for the Future. (1982). Alliance for progress, a social invention in the making. Baltimore [Md.: Published for Resources for the Future by Johns Hopkins Press.

PRATT, M. L. (2011). Ojos imperiales: literatura de viajes y transculturación. Argentina: Fondo de Cultura Económica. 
QUESADA, F. (2010). La marea del Pacífico. La Fundación Ford en Chile (1963-1973). En Autonomía y dependencia académica. Universidad e investigación científica en un circuito periférico: Chile y Argentina (1950-1980) (pp. 89-102). Buenos Aires: Biblos.

ROBIN, J. P., \& TERZO, F. C. (1972). Urbanization in Chile (No. 002013) (p. 68). New York: Ford Foundation. Recuperado de Rockefeller Archive Center. (FA739A - Box 79)

ROBIN, J. P., TERZO, F. C., \& VALENZUELA SHOLZ, J. (1972). Urbanization in Venezuela (No. 002012) (p. 120). New York: Ford Foundation. Recuperado de Rockefeller Archive Center. (FA739A - Box 79)

TERZO, F. C. (1972). Urbanization in the Developing Countries: the response of International Assistance (No. 002022) (p. 133). New York: Ford Foundation. Recuperado de Rockefeller Archive Center. (FA739A - Box 80) 